\title{
„Ehelicher Güterstand“: welche Fragen werden von der Verordnung erfasst, welche fallen nicht in den Anwendungsbereich?
}

\section{"Matrimonial Property regime": which subjects are covered under this term in the Matrimonial Property Regulation?}

\author{
Barbara Reinhartz ${ }^{1}$ \\ Online publiziert: 2. August 2019 \\ (C) The Author(s) 2019
}

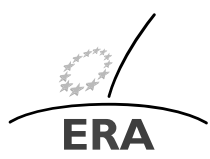

EUROPÄISCHE RECHTSAKADEMIE ACADEMY OF EUROPEAN LAW ACADEMIE DE DROIT EUROPEEN ACCADEMIA DI DIRITTO EUROPEO TRIER - TREVES - TREVIRI

Zusammenfassung In diesem Beitrag wird der Anwendungsbereich der Ehegüterrechtsverordnung besprochen.

Dabei werden die folgende Aspekte näher erörtert:

1. die Abgrenzung der Ehegüterverordnung zur Partnerschaftsverordnung, wobei die Frage: „was ist eine Ehe?” und die Anerkennung von ausländischen Ehen von gleichgeschlechtlichen Paaren im Vordergrund steht;

2. die Abgrenzung zur Erbrechtsverordnung, wobei die Mahnkopf-Entscheidung natürlich nicht fehlen darf;

3. die Frage, in wieweit das régime primaire, das heißt: die allgemeinen Ehewirkungen, unter die Ehegüterverordnung fällt; und

4. die übrigen Abgrenzungen im Vergleich zur Erbrechtverordnung.

Abstract In this paper on the scope of application of the EU Regulation on matrimonial property the following questions will be discussed:

1. What is a "marriage" in the context of the Regulation?

2. Where is the division between matrimonial property law and succession law according to the Mahnkopf case?

3. How is the régime primaire covered by the Matrimonial Property Regulation? Via application of the lex causae and/or via art. 30 of the Regulation?

4. Are the other excluded subjects the same as in the Succession Regulation?

Schlüsselwörter EU-Ehegüterrechtsverordnung · Anwendungsbereich · Allgemeine Ehewirkungen · Anerkennung gleichgeschlechtlicher Ehen

Dieser Artikel beruht auf einem Referat der Verfasserin im Rahmen der „Jahrestagung Europäisches Familienrecht" der ERA vom 25./26. Oktober 2018 in Trier.

B. Reinhartz

B.E.Reinhartz@uva.nl

1 Prof. Dr., Universität Amsterdam, Amsterdam, Niederlande 
Keywords EU Matrimonial Property Regulation · Scope · Regime primaire · Recognition of same sex marriages

\section{Einleitung}

Vor elf Jahren war eine EU-Regelung noch Zukunftsmusik. Damals konnte ich nicht mehr als einige Trends besprechen, als ich für die ERA einen Vortrag über das internationale Ehegüterrecht hielt. Inzwischen sind am 29. Januar 2019 in 18 Ländern der EU die EU-Ehegüterrechtsverordnung (EuGüVO) und die EUPartnerschaftsverordnung (EuPartVO) in Kraft getreten.

Mein Thema für diesen Beitrag ist der Anwendungsbereich der Ehegüterrechtsverordnung. Ich werde mich dabei auf die folgenden Aspekte konzentrieren:

1. die Abgrenzung der Ehegüterrechtsverordnung zur Partnerschaftsverordnung, wobei die Frage: ,was ist eine Ehe?“ und die Anerkennung ausländischer Ehen von gleichgeschlechtlichen Paaren im Vordergrund steht;

2. die Abgrenzung zur Erbrechtsverordnung, wobei die Mahnkopf-Entscheidung nicht fehlen darf;

3. die Frage, in wieweit das régime primaire, das heißt: die allgemeinen Ehewirkungen, unter die Ehegüterverordnung fällt; und

4. die übrigen Abgrenzungen zur Erbrechtverordnung.

\section{Was ist eine Ehe?}

Es ist klar, dass die Ehegüterrechtsverordnung das IPR-Güterrecht von Ehegatten umfasst. Dabei muss man sich die Frage stellen: was ist eine Ehe im Sinne der Ehegüterrechtsverordnung? Obwohl die Verordnung nur im Rahmen der engeren $\mathrm{Zu}$ sammenarbeit von 18 Mitgliedstaaten der EU zustande gekommen ist, ${ }^{1}$ stimmt die Auffassung über den Inhalt einer Ehe (oder einer eingetragenen Lebenspartnerschaft, soweit vorhanden) in diesen 18 Staaten sicher nicht überein. Manche Staaten begrenzen die Ehe auf die Verbindung zwischen einem Mann und einer Frau und reservieren die eingetragene Lebenspartnerschaft für gleichgeschlechtliche Paare; andere haben nur die Ehe und keine eingetragene Lebenspartnerschaft, während wiederum andere Staaten die Ehe für alle Paare anbieten. Zum Schluss gibt es auch noch Staaten, die beide Optionen für alle Paare anbieten.

In Europa sehen wir klar den Trend zur Ehe, die auch für Paare gleichen Geschlechts möglich ist, wobei die Entscheidung des EGMR vom 24.6.2010 (Schalk \& Kopf/Österreich $)^{2}$ wohl einigen Druck in die Richtung gegeben hat. ${ }^{3}$ Daher wird

\footnotetext{
${ }^{1}$ Laut Erwägungsgrund 11 handelt es sich um Belgien, Bulgarien, die Tschechische Republik, Deutschland, Griechenland, Spanien, Frankreich, Kroatien, Italien, Luxemburg, Malta, die Niederlande, Österreich, Portugal, Slowenien, Finnland und Schweden und Zypern. Estland wird in absehbarer Zeit wohl auch Mitgliedstaat.

${ }^{2}$ EGMR 24.6.2010, nr. 30141/04 (Schalk \& Kopf/Österreich) [1].

${ }^{3}$ Inzwischen sind viele Mitgliedstaaten der EU den Niederlanden gefolgt, die die Ehe bereits 2001 für Paare gleichen Geschlechts ermöglicht haben: Belgien (2003), Spanien (2005), Schweden (2009), Portugal
} 
in immer mehr Ehen die Frage aktuell, ob eine solche Ehe auch anerkannt werden muss in Mitgliedstaaten der Verordnung, die die Ehe im Moment noch exklusiv für Mann und Frau reservieren. Ein Ausweg, der von manchen abgelehnt wird, ist, dass ein solcher Staat die gleichgeschlechtliche Ehe als eingetragene Lebenspartnerschaft anerkennt. ${ }^{4}$ Erwägungsgrund 38 gibt einen anderen Ausweg für Probleme bei der gerichtlichen Zuständigkeit in diesem Fall:

„Die Gerichte eines Mitgliedstaats können feststellen, dass nach ihrem Internationalen Privatrecht die betreffende Ehe für die Zwecke eines Verfahrens über den ehelichen Güterstand nicht anerkannt werden kann. In solchen Fällen kann es ausnahmsweise erforderlich sein, die durch diese Verordnung begründete Zuständigkeit abzulehnen. Die Gerichte sollten rasch handeln, und die betroffene Partei sollte die Möglichkeit haben, die Rechtssache in jedem anderen Mitgliedstaat, dessen gerichtliche Zuständigkeit aufgrund eines Anknüpfungspunkts begründet ist, anhängig zu machen, wobei es nicht auf die Rangfolge der Zuständigkeitskriterien ankommt und zugleich die Parteiautonomie zu wahren ist. Jedes nach einer Unzuständigkeitserklärung angerufene Gericht, das nicht ein Gericht des Mitgliedstaats ist, in dem die Ehe geschlossen wurde, darf sich unter denselben Bedingungen ebenfalls ausnahmsweise für unzuständig erklären. Eine Kombination der verschiedenen Zuständigkeitsregeln sollte jedoch gewährleisten, dass die Parteien jede Möglichkeit haben, ein Gericht eines Mitgliedstaats anzurufen, das sich zu dem Zweck, ihrem ehelichen Güterstand Wirkung zu verleihen, für zuständig erklärt.“

Auf diese Weise wird den Mitgliedstaaten, die große Probleme mit der gleichgeschlechtlichen Ehe haben, auch ein Ausweg auf dem Gebiet der Zuständigkeit geboten. Die Europäische Kommission wollte auf jeden Fall vermeiden, dass die Anerkennung einer gleichgeschlechtlichen Ehe über den Umweg des ordre public verweigert werden könnte, weil dies gegen Artikel 21 der Charta der Grundrechte der Europäischen Union, der den Grundsatz der Nichtdiskriminierung enthält, verstoßen würde. 5

\section{Die Abgrenzung zur Erbrechtsverordnung}

Vor allem im deutschen Recht stellte sich jahrzehntelang die Frage, wie § 1371 BGB und $\S 1931$ Absatz 4 BGB im Sinne der Verordnung qualifiziert werden sollten. Es sah danach aus, dass $\S 1371$ Absatz 1 BGB $^{6}$ als ehegüterrechtlich qualifiziert werden

(2010), Dänemark (2012), Frankreich (2013), Großbritannien (2014) und zuletzt Luxemburg und Irland (2015). Vergleiche C.M. Waaldijk, Ars Aequi 2016, 237-248, https://openaccess.leidenuniv.nl/bitstream/ handle/1887/45697/Waaldijk-15jaaropenstellinghuwelijk-inArsAequi-april2016.pdf?sequence=1 [2].

${ }^{4}$ Johannes Weber, Die Europäischen Güterrechtsverordnungen: Eine erste Annäherung, DNotZ 2016, 669 [3].

${ }^{5}$ Siehe auch Erwägungsgrund 54.

${ }^{6}$ Wird der Güterstand durch den Tod eines Ehegatten beendet, so wird der Ausgleich des Zugewinns dadurch verwirklicht, dass sich der gesetzliche Erbteil des überlebenden Ehegatten um ein Viertel der Erbschaft erhöht; hierbei ist unerheblich, ob die Ehegatten im einzelnen Falle einen Zugewinn erzielt haben. 
müsse. So urteilte der BGH noch im Mai $2015,{ }^{7}$ obwohl unumstritten war, dass dieses extra Viertel im europäischen Erbschein vermerkt werden kann. ${ }^{8}$

$\S 1931$ Absatz 4 BGB gehörte anderseits ${ }^{9}$ nach herrschender Meinung zum Erbrecht und folglich - so wurde angekommen - zum Anwendungsbereich der Erbrechtsverordnung. ${ }^{10}$ Dies war die Folge des deutschen Systems, das die erbrechtliche Position des überlebenden Ehegatten mit dem Ehegüterrecht verknüpft, das zwischen den Ehepartnern zu Lebzeiten galt. In vielen anderen Ländern gibt es diese Verknüpfung nicht.

Am 1. März 2018 schuf der EuGH in der Mahnkopf-Entscheidung Klarheit in dieser Frage. Er entschied - anders als der BGH -, dass beide Regelungen erbrechtlicher Natur sind und daher in den Anwendungsbereich der Erbrechtverordnung fallen. ${ }^{11}$ Damit sind sie vom Anwendungsbereich der Ehegüterrechtsverordnung ausgeschlossen.

\section{Allgemeine Ehewirkungen}

Als drittes kann man sich die Frage stellen, in wieweit die allgemeinen Ehewirkungen (international auch als régime primaire bezeichnet), unter den Anwendungsbereich der Ehegüterrechtsverordnung fallen. Man kann dabei an die Möglichkeit denken, dass der Mann der Frau vorschreiben kann, dass sie nicht einer Erwerbstätigkeit nachgehen darf. Oder an die Frage, in wieweit der eine Ehegatte für gewisse Schulden haftet, die der andere Ehegatte eingegangen ist. Oder man kann an den Schutz denken, den das Gesetz dem einen Ehegatten gegen gefährliche Vertragsabschlüsse des anderen Ehegatten gewährt, z.B. im deutschen Recht bei der Zugewinngemeinschaft nach $\S 1365$ BGB, Zustimmung bei Verfügungen über das Vermögen im Ganzen; im niederländischen Recht nach Artikel 1:88 BW, der u.a. Zustimmung zum Verkauf der Ehewohnung und anderen Verträgen, die das Ende des Wohnrechts an der Ehewohnung bedeuten können, zu den meisten Schenkungen, zu Bürgschaften und vergleichbaren Verträgen vorschreibt, wobei man für Schulden eines Dritten, und für Ratenkaufverträge aufkommen muss. Ich werde mich heute auf die finanziellen Ehewirkungen beschränken.

\footnotetext{
${ }^{7}$ BGH 13.5.2015 - IV ZB 30/14, BGHZ 205, 289, Rz. 24 ff; FamRZ 2015, 1180 m. Anm. Mankowski [4]. Dutta, Das neue internationale Güterrecht der Europäischen Union - ein Abriss der europäischen Güterrechtsverordnungen, FamRZ 2016, 1974 [5].

${ }^{8}$ MünchKomm/Dutta, BGB, 2015, Art. 63 EuErbVO, Rz. 8; Johannes Weber, Die Europäischen Güterrechtsverordnungen: Eine erste Annäherung, DNotZ 2016, 666 [6].

${ }^{9}$ Bestand beim Erbfall Gütertrennung und sind als gesetzliche Erben neben dem überlebenden Ehegatten ein oder zwei Kinder des Erblassers berufen, so erben der überlebende Ehegatte und jedes Kind zu gleichen Teilen $(\ldots)$.

${ }^{10}$ J.P. Schmidt in Dutta/Weber, Internationales Erbrecht, 2016, Art. 1 EuErbVO, Rz 49 [7]; Johannes Weber, Interdependenzen zwischen Europäischer Erbrechtsverordnung und Ehegüterrecht - de lege lata und de lege ferenda, DNotZ 2016, 434 mit einigen Beispielen aus verschiedenen europäischen Rechtssystemen; Johannes Weber, Die Europäischen Güterrechtsverordnungen: Eine erste Annäherung, DNotZ 2016, $666[3]$.

${ }^{11}$ C-558/16, ECLI:EU:C:2018:138, § 40-44. Die Entscheidung ist im Material aufgenommen in der Rubrik Entscheidungen zur Erbrechtsverordnung unter Nr. 10.
} 
Unter den Anwendungsbereich der Verordnung fällt das Thema der Haftung für gewisse Schulden des anderen Ehegatten, so bestimmt Art. 27 EuGüVO:

„Das nach dieser Verordnung auf den ehelichen Güterstand anzuwendende Recht regelt unter anderem

(...)

c) die Haftung des einen Ehegatten für die Verbindlichkeiten und Schulden des anderen;

$(\ldots)^{\star}$

Schwieriger wird es, wenn wir uns die Materie des $\S 1365$ BGB oder des Artikels 1:88 BW ansehen. Diese Ehewirkungen sind anderer Art. Wie sind diese zu qualifizieren?

Etwas mehr Klarheit geben die Erwägungsgründe 18-20:

„(18) Der Anwendungsbereich dieser Verordnung sollte sich auf alle zivilrechtlichen Aspekte der ehelichen Güterstände erstrecken und sowohl die Verwaltung des Vermögens der Ehegatten im Alltag betreffen als auch die güterrechtliche Auseinandersetzung, insbesondere infolge der Trennung des Paares oder des Todes eines Ehegatten. Für die Zwecke dieser Verordnung sollte der Begriff ,ehelicher Güterstand“ autonom ausgelegt werden und er sollte nicht nur Regelungen umfassen, von denen die Ehegatten nicht abweichen dürfen, sondern auch fakultative Regelungen, die sie nach Maßgabe des anzuwendenden Rechts vereinbaren können, sowie die Auffangregelungen des anzuwendenden Rechts. Dieser Begriff schließt nicht nur vermögensrechtliche Regelungen ein, die bestimmte einzelstaatliche Rechtsordnungen speziell und ausschließlich für die Ehe vorsehen, sondern auch sämtliche vermögensrechtlichen Verhältnisse, die zwischen den Ehegatten und in ihren Beziehungen gegenüber Dritten direkt infolge der Ehe oder der Auflösung des Eheverhältnisses gelten.

(19) Aus Gründen der Klarheit sollte eine Reihe von Fragen, die als mit dem ehelichen Güterstand zusammenhängend betrachtet werden könnten, ausdrücklich vom Anwendungsbereich dieser Verordnung ausgenommen werden.

(20) Dementsprechend sollte diese Verordnung nicht für Fragen der allgemeinen Rechts-, Geschäfts- und Handlungsfähigkeit der Ehegatten gelten; dieser Ausschluss sollte sich jedoch nicht auf die spezifischen Befugnisse und Rechte eines oder beider Ehegatten - weder im Verhältnis untereinander noch gegenüber Dritten - im Zusammenhang mit dem Vermögen erstrecken, da diese Befugnisse und Rechte in den Anwendungsbereich dieser Verordnung fallen sollten.“

Wenn man die Erwägungsgründe 19 und 20 im Zusammenhang liest, sieht es danach aus, dass Nr. 19 sagt, dass diese Schutzregelungen nicht unter den Anwendungsbereich fallen, während Nr. $20 \mathrm{im}$ Gegenteil zu sagen scheint, dass diese Regelungen wohl unter den Anwendungsbereich fallen.

Um die Sache noch komplizierter zu machen, finden wir ganz woanders noch eine Verweisung zu gewissen Aspekten des régime primaire, nämlich in Erwägungsgrund 53, der Artikel 30 EuGüVO näher erklärt: 
„(53) Aus Gründen des öffentlichen Interesses wie der Wahrung der politischen, sozialen oder wirtschaftlichen Ordnung eines Mitgliedstaats sollte es gerechtfertigt sein, dass die Gerichte und andere zuständige Behörden der Mitgliedstaaten die Möglichkeit erhalten, in Ausnahmefällen auf der Grundlage von Eingriffsnormen Ausnahmeregelungen anzuwenden. Dementsprechend sollte der Begriff "Eingriffsnormen" Normen von zwingender Natur wie zum Beispiel die Normen zum Schutz der Familienwohnung umfassen. Diese Ausnahme von der Anwendung des auf den ehelichen Güterstand anzuwendenden Rechts ist jedoch eng auszulegen, damit sie der allgemeinen Zielsetzung dieser Verordnung nicht zuwiderläuft."“

In diesem Erwägungsgrund wird als Beispiel für eine ehegüterrechtliche Eingriffsnorm der Schutz der Familienwohnung genannt. ${ }^{12}$ Im deutschen Recht kann $§ 1365$ BGB angewendet werden, wenn die Familienwohnung einen so großen Teil des Vermögens des Ehegatten umfasst, dass $\S 1365$ BGB anwendbar ist, aber $\S 1365$ BGB umfasst viel mehr als nur den Verkauf der Familienwohnung, denn auch andere Verträge können das Vermögen im Ganzen betreffen, sodass nur noch 10-15\% des Vermögens übrig bleibt.

In den Niederlanden umfasst Artikel 1:88 Absatz 1 unter a BW zwar ausdrücklich den Verkauf der Familienwohnung und andere Fälle, wodurch der andere Ehegatte die Familienwohnung nicht mehr nutzen kann, aber daneben bedürfen noch ganz andere Verträge die Zustimmung des anderen Ehegatten.

Kann man diese Materie, die viel umfangreicher ist als nur die Familienwohnung betreffend, unter diese Eingriffsnorm bringen? Letztendlich wird der EuGH diese Frage entscheiden müssen, aber bevor es soweit ist, wird voraussichtlich noch einige Zeit vergehen. Was kann man jetzt schon dazu sagen? In den Niederlanden wird der ganze Artikel 1:88 BW in IPR-Fällen unter Artikel 30 Ehegüterverordnung begriffen, wie der neue Absatz 7 von Artikel 1:88 BW ab dem 29.1.2019 bestimmt. In dem neuen Absatz steht, dass Artikel 1:88 BW in allen Fällen anwendbar ist, in denen der zu schützende Ehegatte in den Niederlanden seinen gewöhnlichen Aufenthalt (gewone verblijfplaats) hat in dem Moment, in dem der zustimmungsbedürftige Vertrag geschlossen wird; also ganz klar eine Eingriffsnorm im Sinne des Artikels 30 der Verordnung. In den Materialien zum Gesetz wird nicht diskutiert, ob das eigentlich zulässig ist. Ich frage mich, ob dies rechtmäßig ist. Kann man all diese verschiedenen Regeln, die dem Schutz der Familie und/oder des anderen Ehegatten dienen sollen, dieser Eingriffsnorm zuordnen? Es scheint mir eher, dass diese Schutznormen zum Anwendungsbereich der Verordnung gehören, wie auch Erwägungsgrund 20 nahelegt. In den Niederlanden ist die Qualifikation als Eingriffsnorm auch umstritten, aber allgemein wird angenommen, dass diese Schutznormen unter beide Bereiche gebracht werden können. Der Schutz der Ehewohnung in Erwägungsgrund 53 ist ganz

\footnotetext{
${ }^{12}$ Vgl. für Belgien: Art. $215 \S 1$ Code civil; für Bulgarien: Art. 34, 26 Familiengesetzbuch; für Finnland: Art. 38 Ehegesetz (mit der Möglichkeit des gutgläubigen Erwerbs); für Frankreich: Art. 215 Abs. 3 Code civil; für Kroatien: Art. 32 Abs. 3 Familiengesetz; für Litauen: Art. 3.36 Abs. 2 ZGB (mit der Möglichkeit eines wirksamen Erwerbs bei fehlender Registrierung als Familienvermögen); für die Niederlande: Art. 1:88 Burgerlijk Wetboek (BW); für die Schweiz: Art. 169 ZGB; für Spanien: Art. 1320 Código Civil (mit der Möglichkeit des gutgläubigen Erwerbs bei falschen Angaben des Veräußerers); für die Türkei: Art. 194 ZGB. (übernommen aus Johannes Weber, Sachenrecht und Verkehrsschutz aus der Perspektive der Europäischen Güterrechtsverordnungen, RNotZ 2017, 365, 369 [8]).
} 
klar nur als Beispiel genannt; andere Schutzregelungen sollten damit sicher nicht ausgeschlossen werden.

In Deutschland liegt es näher, $\S 1365$ BGB als ehegüterrechtlich zu qualifizieren, da diese Regelung nur gilt, wenn zwischen den Ehegatten die Zugewinngemeinschaft gilt. Gilt dagegen Gütertrennung, dann ist $\S 1365$ BGB nicht anwendbar. Johannes Weber plädiert in der Rheinischen Notar-Zeitschrift vom letzten Jahr dafür, ${ }^{13}$ auch die Verfügungsbeschränkungen, die unabhängig vom Güterstand gelten, wegen Artikel 27 lit. f der Verordnung unter den Anwendungsbereich der Ehegüterverordnung zu bringen, da sie Folgen für das Rechtsverhältnis zwischen einem Ehegatten und einem Dritten haben. Diese Lösung erscheint mir richtiger als die, welche in den Niederlanden gewählt ist, ${ }^{14}$ aber es wird sich zeigen, wie der EuGH diese Beschränkungen qualifiziert. ${ }^{15}$

\section{Die übrigen Abgrenzungen im Vergleich zur Erbrechtsverordnung}

Außer den Abgrenzungsproblemen, die die Ehegüterrechtsverordnung im Besonderen mit sich bringt, sehen wir in Artikel 1 der Verordnung einige Teilbereiche des Rechts ausgeschlossen, die auch von der Erbrechtsverordnung ausgeschlossen sind. Da schon viel über die Erbrechtsverordnung geschrieben wurde und auch schon die ersten EuGH-Entscheidungen bekannt werden, werde ich diese Materie nur kurz besprechen.

In Absatz 1 werden Steuer- und Zollsachen sowie verwaltungsrechtliche Angelegenheiten ausgeschlossen. Das kennen wir schon von der Erbrechtsverordnung.

In Absatz 2 werden ausgeschlossen

a) die Rechts-, Geschäfts- und Handlungsfähigkeit der Ehegatten;

b) das Bestehen, die Gültigkeit oder die Anerkennung einer Ehe;

c) die Unterhaltspflichten;

d) die Rechtsnachfolge nach dem Tod eines Ehegatten;

e) die soziale Sicherheit;

f) die Berechtigung, Ansprüche auf Alters- oder Erwerbsunfähigkeitsrente, die während der Ehe erworben wurden und die während der Ehe zu keinem Renteneinkommen geführt haben, im Falle der Ehescheidung, der Trennung ohne Auflösung des Ehebands oder der Ungültig Erklärung der Ehe zwischen den Ehegatten zu übertragen oder anzupassen;

g) die Art der dinglichen Rechte an Vermögen, und

h) jede Eintragung von Rechten an beweglichen oder unbeweglichen Vermögensgegenständen in ein Register, einschließlich der gesetzlichen Voraussetzungen für

\footnotetext{
${ }^{13}$ Johannes Weber, Sachenrecht und Verkehrsschutz aus der Perspektive der Europäischen Güterrechtsverordnungen, RNotZ 2017, 365.

${ }^{14}$ Diese Lösung habe ich auch vertreten im internationalen Kommentar zur Ehegüterverordnung, den ich mit Ulf Bergquist, Richard Frimston, Paul Lagarde und Domenico Damascelli geschrieben habe. Der Kommentar erschien Anfang 2019 bei Oxford University Press auf Englisch; eine französische Fassung ist bei Dalloz erschienen.

${ }^{15}$ Siehe dazu ausführlicher meinen Beitrag in einem Special des WPNR (Weekblad voor Privaatrecht, Notariaat en Registratie), 2018/7216, 900-906 [9].
} 
eine solche Eintragung, sowie die Wirkungen der Eintragung oder der fehlenden Eintragung solcher Rechte in ein Register.“

Diese Ausschlüsse haben ihren Grund oft darin, dass die Materie schon in einem anderen Übereinkommen geregelt ist:

Das Bestehen, die Gültigkeit oder die Anerkennung einer Ehe (lit. b) werden im CIEC-Übereinkommen über die Anerkennung von Entscheidungen in Ehesachen von 1967 geregelt. $^{16}$

Der Ausschluss der Unterhaltspflichten unter c) ist evident, da es dafür andere EU-Instrumente gibt: die EU Unterhaltsverordnung 4/2009 und das Haager Unterhaltsprotokoll 2007. Der Ausschluss bezieht sich sowohl auf Unterhalt während der Ehe als auch danach.

Die Rechtsnachfolge im Falle des Todes eines Ehegatten wird in der EU Erbrechtverordnung 650/2012 geregelt. $^{17}$

Die soziale Sicherheit ist eine interne Angelegenheit der Mitgliedsstaaten.

Die Frage der Ansprüche auf Alters- oder Erwerbsunfähigkeitsrente in bestimmten Fällen ist insofern kompliziert, als dieses Thema in den Mitgliedstaaten sehr unterschiedlich geregelt ist. ${ }^{18}$ In den Niederlanden zum Beispiel sparen die meisten Angestellten und Beamten für die Altersrente, die nicht aus dem laufenden Budget des Staates gezahlt wird, neben einer bescheidenen staatlichen Altersrente (AOW). Dutta $^{19}$ nimmt an, dass in manchen Fällen diese Altersrenten, die die Ehegatten aus dem Einkommen gespart haben, doch zum Güterrecht der Ehegatten gerechnet werden sollten (siehe auch Erwägungsgrund 23). In den Niederlanden kennen wir diesen Gedanken auch, wenn es darum geht, ob bestimmte Frührenten in die Gütergemeinschaft fallen oder nicht. ${ }^{20}$

Die Ehegüterrechtsverordnung beschäftigt sich auch nicht mit der Art der dinglichen Rechte, da dies eine Aufgabe der Staaten ist (siehe lit. g). Falls das anzuwendende Recht gewisse dingliche Rechte kennt, die in einem anderen Staat unbekannt sind, kann über die Anpassung dieses Rechts zu einem praktisch akzeptablen Ergebnis gelangt werden. Eventuelle Eigentumsverhältnisse z.B. an Grundstücken müssen danach auch im Grundbuch vermerkt werden können. Die Eintragung von im Lande unbekannten ausländischen Eigentumsverhältnissen ist im Allgemeinen kaum möglich (lit. h).

In den Erwägungsgründen 24-28 wird dieses näher erklärt:

\footnotetext{
${ }^{16}$ https://www.personenstandsrecht.de/Webs/PERS/DE/uebereinkommen/_documents/ciec/ue11.html; jsessionid=83641D848A8A3AB675FBE9E8585C295A.2_cid295 [10].

${ }^{17}$ Richard Frimston, in: Commentary on the EU Regulation on Succession and Wills, Sellier/Otto Schmidt Verlag 2015 by Bergquist, Damascelli, Frimston, Lagarde, Odersky and Reinhartz, Art. 1 Rz 25-30 [11].

${ }^{18}$ In Deutschland gibt es den Versorgungsausgleich (auf den Artikel 17 Abs. 3 EGBGB anwendbar bleibt); in den Niederlanden gibt es die Pensioenverevening (geregelt in Artikel 10:51 BW und Artikel 1 Absatz 7 Wet verevening pensioenrechten bij scheiding, die anwendbar bleiben). Rainer Hausmann, Die Inhaltskontrolle von Eheverträgen in Fällen mit Auslandsberührung, in Fairness, Justice, Equity, Festschrift Geimer 2017, 207-208 [12].

${ }^{19}$ Dutta, Das neue internationale Güterrecht der Europäischen Union - ein Abriss der europäischen Güterrechtsverordnungen, FamRZ 2016, 1975.

${ }^{20}$ Tekst en Commentaar Personen- en familierecht, aant. 9 bij art. 1 WVPS; Rechtbank Zutphen 22.3.2006, ECLI:NL:RBZUT:2006:AV9123, JPF 2006/85, m. Anmerkung Reinhartz [14].
} 
„(24) Diese Verordnung sollte die sich aus dem ehelichen Güterstand ergebende Begründung oder Übertragung eines Rechts an beweglichen oder unbeweglichen Vermögensgegenständen nach Maßgabe des auf den ehelichen Güterstand anzuwendenden Rechts ermöglichen. Sie sollte jedoch nicht die abschließende Anzahl (Numerus clausus) der dinglichen Rechte berühren, die das nationale Recht einiger Mitgliedstaaten kennt. Ein Mitgliedstaat sollte nicht verpflichtet sein, ein dingliches Recht an einer in diesem Mitgliedstaat belegenen Sache anzuerkennen, wenn sein Recht dieses dingliche Recht nicht kennt.

(25) Damit die Ehegatten jedoch die Rechte, die durch den ehelichen Güterstand begründet worden oder auf sie übergegangen sind, in einem anderen Mitgliedstaat ausüben können, sollte diese Verordnung die Anpassung eines unbekannten dinglichen Rechts an das in der Rechtsordnung dieses anderen Mitgliedstaats am ehesten vergleichbare Recht vorsehen. Bei dieser Anpassung sollten die mit dem besagten dinglichen Recht verfolgten Ziele und Interessen und die mit ihm verbundenen Wirkungen berücksichtigt werden. Für die Zwecke der Bestimmung des am ehesten vergleichbaren dinglichen Rechts können die Behörden oder zuständigen Personen des Staates, dessen Recht auf den ehelichen Güterstand Anwendung findet, kontaktiert werden, um weitere Auskünfte zu der Art und den Wirkungen des betreffenden Rechts einzuholen. In diesem Zusammenhang könnten die bestehenden Netze im Bereich der justiziellen Zusammenarbeit in Zivil- und Handelssachen sowie die anderen verfügbaren Mittel, die die Erkenntnis ausländischen Rechts erleichtern, genutzt werden.

(26) Die in dieser Verordnung ausdrücklich vorgesehene Anpassung unbekannter dinglicher Rechte sollte andere Formen der Anpassung im Zusammenhang mit der Anwendung dieser Verordnung nicht ausschließen.

(27) Die Voraussetzungen für die Eintragung von Rechten an beweglichen oder unbeweglichen Vermögensgegenständen in ein Register sollten vom Anwendungsbereich dieser Verordnung ausgenommen werden. Somit sollte das Recht des Mitgliedstaats, in dem das Register geführt wird (für unbewegliches Vermögen das Recht der belegenen Sache (lex rei sitae), bestimmen, unter welchen gesetzlichen Voraussetzungen und wie die Eintragung vorzunehmen ist und welche Behörden wie etwa Grundbuchämter oder Notare dafür zuständig sind zu prüfen, dass alle Eintragungsvoraussetzungen erfüllt sind und die vorgelegten oder erstellten Unterlagen vollständig sind beziehungsweise die erforderlichen Angaben enthalten. Insbesondere können die Behörden prüfen, ob es sich bei dem Recht eines Ehegatten an dem Vermögensgegenstand, der in dem für die Eintragung vorgelegten Schriftstück erwähnt ist, um ein Recht handelt, das als solches in dem Register eingetragen ist oder nach dem Recht des Mitgliedstaats, in dem das Register geführt wird, anderweitig nachgewiesen wird. (...)

(28) Die Wirkungen der Eintragung eines Rechts in ein Register sollten ebenfalls vom Anwendungsbereich dieser Verordnung ausgenommen werden. Daher sollte das Recht des Mitgliedstaats, in dem das Register geführt wird, dafür 
maßgebend sein, ob beispielsweise die Eintragung deklaratorische oder konstitutive Wirkung hat. Wenn also zum Beispiel der Erwerb eines Rechts an einer unbeweglichen Sache nach dem Recht des Mitgliedstaats, in dem das Register geführt wird, die Eintragung in ein Register erfordert, damit die Wirkung ,erga omnes“ von Registern sichergestellt wird oder Rechtsgeschäfte geschützt werden, sollte der Zeitpunkt des Erwerbs dem Recht dieses Mitgliedstaats unterliegen.“

Ein konkretes Beispiel ist die Gütergemeinschaft des niederländischen Rechts. Diese Kombination von vollem Eigentum beider Ehegatten in Bezug auf die Güter, die zur Gütergemeinschaft gehören, gibt es in Deutschland meines Wissens in dieser Form nicht. ${ }^{21}$ Zwar gibt es seit dem zum 1.1.2018 geänderten Ehegüterrecht ${ }^{22}$ nunmehr eine Errungenschaftsgemeinschaft in den Niederlanden, aber die alten Gütergemeinschaften von vor 2018 bleiben unverändert bestehen, soweit sie nicht durch die Ehepaare in einem Ehevertrag geändert werden. In beiden Fällen bleibt, so die herrschende Meinung, die Auffassung über das Wesen der Gütergemeinschaft - sei sie größer nach altem Recht oder kleiner nach neuem Recht - unverändert.

\section{Zum Schluss}

Neue Regelungen führen immer zu neuen Fragen, die erst in den danach folgenden Jahren oder Jahrzehnten beantwortet werden. Insofern kann ich mir vorstellen, dass manche Praktiker wegen der mit der neuen Verordnung verbundenen Rechtsunsicherheit vorläufig etwas weniger glücklich sind. Anderseits gehe ich davon aus, dass durch die Harmonisierung der gerichtlichen Zuständigkeit, der Regeln des anwendbaren Rechts, die Anerkennung von Eheverträgen und die Regeln der Abwicklung der Ehegüterstände so viel gewonnen ist, dass die durch das Inkrafttreten der Verordnung entstehenden Nachteile der Verordnung mehr als kompensiert werden. Alles zusammengenommen sind wir froh, dass die europäische Ehegüterechtsverordnung endlich in Kraft getreten ist für die Ehepaare, die ab dem 29. Januar 2019 heiraten oder das anwendbare Recht auf ihr Vermögen ändern.

Hinweis des Verlags Der Verlag bleibt in Hinblick auf geografische Zuordnungen und Gebietsbezeichnungen in veröffentlichten Karten und Institutsadressen neutral.

Open Access This article is distributed under the terms of the Creative Commons Attribution 4.0 International License (http://creativecommons.org/licenses/by/4.0/), which permits unrestricted use, distribution, and reproduction in any medium, provided you give appropriate credit to the original author(s) and the source, provide a link to the Creative Commons license, and indicate if changes were made.

${ }^{21}$ Johannes Weber, Die Europäischen Güterrechtsverordnungen: Eine erste Annäherung, DNotZ 2016, 667; Rainer Hausmann, Internationales und Europäisches Ehescheidungsrecht, 2013, F, Rz 106-108.

${ }^{22}$ Gesetzentwurf 33 987, der in gewissen Teilen dem ursprünglichen Text des Gesetzentwurfs 28867 gleicht, der 2012 stark geändert in Kraft trat, ist zum Jahresbeginn 2018 in Kraft getreten. Im Allgemeinen gelten die neuen Regeln nur für Gütergemeinschaften, die seit dem Datum entstanden sind. 


\section{Literatur}

1. Schalk \& Kopf/Österreich: EGMR 24.06.2010, Nr. 30141/04

2. Waaldijk, C.M.: Ars Aequi, 237-248 (2016). https://openaccess.leidenuniv.nl/bitstream/handle/1887/ 45697/Waaldijk-15jaaropenstellinghuwelijk-inArsAequi-april2016.pdf?sequence=1

3. Weber, J.: Die Europäischen Güterrechtsverordnungen: Eine erste Annäherung. DNotZ (2016)

4. BGH 13.5.2015 - IV ZB 30/14, BGHZ 205, 289, Rz. 24 ff; FamRZ (2015), 1180, m. Anm. Mankowski

5. Dutta, A.: Das neue internationale Güterrecht der Europäischen Union - ein Abriss der europäischen Güterrechtsverordnungen, FamRZ 2016 (1974)

6. MünchKomm/Dutta, BGB (2015), Art. 63 EuErbVO, Rz. 8

7. Schmidt, J.P.: In: Dutta/Weber: Internationales Erbrecht (2016). Art. 1 EuErbVO, Rz 49

8. Weber, J.: Sachenrecht und Verkehrsschutz aus der Perspektive der Europäischen Güterrechtsverordnungen. RNotZ (2017), 365, 369

9. Weekblad voor Privaatrecht, Notariaat en Registratie, 2018/7216

10. Bundesministerium des Innern, für Bau und Heimat. https://www.personenstandsrecht.de/Webs/ PERS/DE/uebereinkommen/_documents/ciec/ue11.html;jsessionid= 83641D848A8A3AB675FBE9E8585C295A.2_cid295

11. Frimston, R.: In: Commentary on the EU Regulation on Succession and Wills, Sellier/Otto Schmidt Verlag (2015), by Bergquist, Damascelli, Frimston, Lagarde, Odersky and Reinhartz, Art. 1 Rz 25-30

12. Hausmann, R.: Die Inhaltskontrolle von Eheverträgen in Fällen mit Auslandsberührung, in Fairness, Justice, Equity, Festschrift Geimer (2017)

13. Tekst en Commentaar, Personen- en familierecht, aant. 9 bij art. 1 WVPS

14. Rechtbank Zutphen 22.3.2006, ECLI:NL:RBZUT:2006:AV9123, JPF 2006/85, m. Anmerkung Reinhartz 